\title{
SUPERFLUIDITY IN NEUTRON STARS
}

\author{
GEORGE GREENSTEIN \\ Amherst College, Amherst, Mass., U.S.A.
}

\begin{abstract}
We present a short Cook's tour of the possible effects of rotation coupled with superfluid properties of neutron star interiors. A suggestion is made to take advantage of forthcoming lunar occultations of the Crab Nebula in order to search for blackbody X-ray emission from the Crab pulsar.
\end{abstract}

\section{Introduction}

Let me begin by summarizing our beliefs (this is the correct term) concerning the internal structure of neutron stars. (Extensive reviews, with references, have been given by Ruderman, 1969a, 1972; Pines, 1970; and Cameron, 1970.) As one penetrates from the surface of a neutron star inwards the density rises rapidly, the electrons quickly become degenerate, and the atoms completely ionized. The nuclei arrange themselves into a rigid lattice. At densities of about $3 \times 10^{11} \mathrm{~g} \mathrm{~cm}^{-3}$ neutrons evaporate from the nuclei. Beneath this point the lattice of nuclei coexists with two uniform gases; a relativistic degenerate gas of electrons and a non-relativistic degenerate gas of neutrons. The number of neutrons outside the nuclei is greater than the number within them. At densities of about $2 \times 10^{14} \mathrm{~g} \mathrm{~cm}^{-3}$ the nuclei dissolve. Beneath this point we have three uniform degenerate gases; relativistic electrons, non-relativistic neutrons and non-relativistic protons. In this regime too the neutrons outnumber the protons. At densities above about $10^{15} \mathrm{~g} \mathrm{~cm}^{-3}$ hyperons appear and rapidly become the dominant component.

We know that at densities below about $2 \times 10^{14} \mathrm{~g} \mathrm{~cm}^{-3}$ the ${ }^{1} S_{\mathrm{o}}$ phase shift between two neutrons is negative; the interaction of two neutrons of opposite spin, in a state of zero relative angular momentum, is attractive. Precisely the same situation holds for electrons in an ordinary metal, although in this case the origin of the attraction is different (in neutron stars the attraction is due to the strong interaction whereas in metals it arises through the electron-phonon interaction). Within the BCS theory of superconductivity the existence of such an attractive interaction constitutes the necessary condition that the electrons become superconducting at sufficiently low temperatures. This result is independent of the origin of the attraction. By analogy, one then reasons that those neutrons in neutron stars for which the ${ }^{1} S_{0}$ phase shift is negative - i.e., those within the crust - form a neutral superconductor - a superfluid.

Near the base of the crust, at $1.6 \times 10^{14} \mathrm{~g} \mathrm{~cm}^{-3}$, the ${ }^{1} S_{\mathrm{o}}$ phase shift becomes positive. At higher densities the ${ }^{3} P_{2}$ phase shift becomes negative, leading to anisotropic superfluidity - a state that has never been observed in the laboratory. In what follows we will treat this anisotropic superfluid in precisely the same way as, the more familiar isotropic superfluid - not necessarily a safe procedure.

The strong interaction between protons is the same as that between neutrons. Thus 
the protons lying below the crust may form a superconductor (isotropic, since the density of protons always remains relatively small).

The picture, then, is this; a neutron star consists of an outer crust, an inner crust coexisting with an isotropic superfluid, an anisotropic superfluid coexisting with an isotropic superconductor, and finally a hyperon core of largely unknown properties. Most of the moment of inertia of the star resides in the superfluid.

Calculated superfluid energy gaps vary with density by a factor of perhaps ten. Resulting transition temperatures then vary from about $10^{9}$ to about $10^{10} \mathrm{~K}$, depending on position within the star. It seems clear that neutron stars cool below $10^{10} \mathrm{~K}$ quite rapidly. It is not clear, though it seems likely, that the Crab and Vela pulsars have cooled far below $10^{9} \mathrm{~K}$. Thus much, but not necessarily all, of the superfluid in those two pulsars lies far below its transition temperature. All older pulsars lie very far below their transition temperatures.

It is often said that neutron stars of sufficiently low mass are entirely solid. This is true but misleading. Such stars do remain solid all the way down to their cores. However, they also contain a superfluid whose moment of inertia exceeds that of the crust. There are, however, neutron stars for which superfluidity is unimportant. These are the most massive stars, which consist almost entirely of hyperons surrounded by thin shells of superfluid and crust. (I am neglecting here the possibility that the neutral hyperons may be superfluid; we will have enough to worry about as it is.)

It is sobering to realize that the whole picture $I$ have drawn has received no direct observational test whatsoever, and only two indirect tests. The first indirect test relates to the observed lack of cool white dwarfs. This phenomenon has been successfully interpreted as resulting from the existence, in the cores of these stars, of the same solid lattice we expect to find in the outer regions of neutron stars. As the lattice cools below its Debye temperature its specific heat drops dramatically. The star then rapidly cools to invisibility (Greenstein, 1969). Our only other piece of confirming observational evidence is the very elegant interpretation, which I will describe below, of the postspeedup behavior of the Crab and Vela pulsars. Two indirect tests do not add up to very much. To be conservative I believe we should keep in the back of our minds the possibility that our picture of neutron star interiors is entirely wrong. If you don't believe me, consider the solar neutrino experiment.

The intense magnetic field pervading the star (the superconductor is not expected to exhibit a Meissner effect) will force the crust, the electrons, the protons (whether superconducting or not) and the charged hyperons to rotate uniformly. The rotation of this 'charged particle system' determines the rate of ticking of the pulsar. The superfluid, however, need not rotate uniformly and, if it does, need not rotate at the same rate as the pulsar. In order to find out what the superfluid is doing we need to understand the ways it has of interacting with the charged particle system.

Because the superfluid is probably far below its transition temperature, these interactions will take place via the superfluid vortex lines. Those neutrons that lie within the normal cores of these lines will scatter against charges. Only one scattering process has been quantitatively considered so far: this is the scattering of neutrons 
in the isotropic superfluid against electrons. Others include scatterings against phonons in the crust, and protons in the cores of proton flux lines. As yet we do not know which of these is the dominant process.

All of these processes lead to a force per $\mathrm{cm}^{3} \mathbf{f}$ between superfluid and charges of the following form

$$
\mathbf{f}=\frac{\varrho_{\mathbf{n}} \varrho_{\mathrm{c}}}{\varrho \tau}\left(\mathbf{v}_{\mathbf{n}}^{\mathrm{e}}-\mathbf{v}_{\mathbf{c}}\right)
$$

where $\varrho_{\mathrm{n}}$ is the mass-energy density of neutrons, $\varrho_{\mathrm{c}}$ that of charges, $\varrho \equiv \varrho_{\mathrm{n}}+\varrho_{\mathrm{c}},\left(\mathbf{v}_{\mathrm{n}}-\mathbf{v}_{\mathrm{c}}\right)$ is the relative velocity between neutrons and charges and $\tau$ has the dimensions of a time. In the absence of external forces acting on the system velocity differences between neutrons and charges are damped out in this time $\tau$. All the physics of the problem is buried in the calculation of $\tau$. If the protons and neutrons are normal $\tau$ is microscopic-something like $10^{-20} \mathrm{~s}$. The rotation of the neutrons is then locked with that of the pulsar. If the protons are normal and the neutrons are superfluid then $\tau$ is on the order of fractions of a second. If the neutrons are superfluid and the protons are superconducting then $\tau$ is increased enormously - one finds values on the order of years.

The pulsar is slowing down. A torque, presumably electromagnetic in origin, acts to decelerate the charges. They are rotating more slowly than the neutrons. If we multiply both sides of (1) by $r \sin \theta$, where $r, \theta$ are the spherical coordinates of the point in question, then the resulting expression gives the torque density acting on the neutrons. Let us assume for now that this is the only torque acting on the neutrons. (In general this is not so.) The velocity $\mathbf{v}_{\mathrm{c}}$ is directed in the $\phi$-direction. We are not assured that the neutrons are rotating in any such simple way. Let us, however, assume for now that the velocities of any bulk circulation currents within the superfluid are small compared with local rotation velocities. Then $\mathbf{v}_{\mathbf{n}}$ is almost in the $\phi$-direction, as is the torque acting on the neutrons. This torque acts to slow their rotation. Equating the torque density to the rate of change of angular momentum yields

$$
\dot{\Omega}_{\mathrm{n}}=\frac{\varrho_{\mathrm{c}}}{\varrho \tau}\left(\Omega_{\mathrm{n}}-\Omega_{\mathrm{c}}\right),
$$

where the $\Omega$ 's are angular velocities. In the steady state $\dot{\Omega}_{\mathrm{n}}=\dot{\Omega}_{\mathrm{c}}$ and we can solve (2);

$$
\frac{\Omega_{\mathrm{n}}-\Omega_{\mathrm{c}}}{\Omega_{\mathrm{c}}}=\frac{\varrho \tau}{\varrho_{\mathrm{c}} t_{\mathrm{c}}}
$$

where $t_{\mathrm{c}} \equiv\left|\Omega_{\mathrm{c}}\right| \dot{\Omega}_{\mathrm{c}} \mid$. So the superfluid is rotating somewhat faster than the pulsar.

\section{The Simplest Possible Model of a Superfluid Neutron Star}

Equations (2) and (3) rest on certain assumptions. For now I wish to consider an approximation within which the assumptions are valid. It consists in considering the 
quantity $\left(\tau \varrho / \varrho_{c}\right)$ constant throughout the star. Then the right-hand side of (3) is constant. Since $\Omega_{\mathrm{c}}$ is constant $\Omega_{\mathrm{n}}$ is also. Therefore the neutrons are rotating uniformly. In this case both assumptions leading to (2) and (3) are valid - the approximation is consistent. There is, however, not the slightest chance that it is correct. I consider it now because the resulting picture is easy to work with and guides our thoughts in fruitful directions. Later on, in the interests of masochism and rigor, the approximation will be relaxed.

\subsection{SPINDOWN FOLLOWING A PERIOD JUMP}

In the first application of these ideas I want to discuss the observed behavior of the Crab and Vela pulsars following their period jumps. So far each of these pulsars has undergone two such jumps and in each case $\Omega_{\mathrm{c}}$ and $\left|\Omega_{\mathrm{c}}\right|$ increased. In a seminal paper Baym et al. (1969) were able to show that, if neutron star interiors are superfluid, an increase in $\Omega_{\mathrm{c}}$ naturally leads to one in $\left|\dot{\Omega}_{\mathrm{c}}\right|$. Their interpretation is elegant and the numerical results too good to be wrong. Their approach is as follows.

Equation (2), after an obvious transformation, now reads

$$
\Omega_{\mathrm{n}}=\frac{I_{\mathrm{c}}}{I \tau}\left(\Omega_{\mathrm{n}}-\Omega_{\mathrm{c}}\right),
$$

where the I's are total moments of inertia. Two torques act on the charges - an accelerating torque equal and opposite to that which decelerates the neutrons and the decelerating radiation torque which we write as $I_{\mathrm{c}} M$. The torque equation for the charges is

$$
\dot{\Omega}_{\mathrm{c}}=-M+\frac{I_{\mathrm{n}}}{I \tau}\left(\Omega_{\mathrm{n}}-\Omega_{\mathrm{c}}\right) .
$$

Now the pulsar speeds up. The accelerating torque in (5) is now less since it is proportional to $\Omega_{\mathrm{n}}-\Omega_{\mathrm{c}}$. The decelerating torque is unchanged. Therefore, as observed, the charges - which constitute the pulsar - decelerate more rapidly. A trivial manipulation of (5) gives the fractional change in $\Omega_{\mathrm{c}}$ divided by that in $\Omega_{\mathrm{c}}$, i.e.,

$$
\zeta \equiv \frac{\Delta \dot{\Omega}_{\mathrm{c}} / \dot{\Omega}_{\mathrm{c}}}{\Delta \Omega_{\mathrm{c}} / \Omega_{\mathrm{c}}}=\frac{I_{\mathrm{n}}}{I} \frac{t_{\mathrm{c}}}{\tau}\left|1-\frac{\Delta \Omega_{\mathrm{n}}}{\Delta \Omega_{\mathrm{c}}}\right| .
$$

There are a number of well-known predictions of this model; the change in $\Omega_{\mathrm{c}}$ decays exponentially away with time constant $\tau$, the instantaneous change in $\Omega_{\mathrm{c}}$ is related to a permanent change in $\Omega_{\mathrm{c}}$ through

where

$$
\left(\Delta \Omega_{\mathrm{c}}\right)_{\text {permanent }}=(1-Q)\left(\Delta \Omega_{\mathrm{c}}\right)_{\mathrm{initial}}
$$

$$
Q=\frac{\tau}{t_{\mathrm{c}}} \tilde{\xi}
$$


and

$$
\frac{\left(\Delta \dot{\Omega}_{\mathrm{c}}\right)^{2}}{\left(\Delta \Omega_{\mathrm{c}}\right)\left(\Delta \ddot{\Omega}_{\mathrm{c}}\right)}=Q .
$$

Most of these predictions are independent of the mechanism producing the period jump.

Because the observed decay times of the Crab and Vela pulsars are long (days and years, respectively) we have strong evidence that these pulsars possess superfluid interiors. It has been claimed that they must also be superconducting. But this is not necessarily so if the coupling time describing the interaction of the superfluid with phonons in the crust is long. If it is then one can imagine that the protons are not superconducting and spin up in fractions of a second following a period jump. The observed period decay would then represent the spin-up of the isotropic superfluid within the crust. To resolve this point we will need a calculation of the phononsuperfluid interaction.

I wish to point out a further testable consequence of this theory. It is that in every model of what caused the period jump, $\xi$ should be constant from one jump to the next (though different for different pulsars). This will be true whether successive jumps have the same or different amplitudes, and whether the time between jumps is long or short compared to the spin-down time $\tau$. It is almost certainly also true within the context of the more realistic model to be described in the next section, although other predictions of the theory (most notably, that of a purely exponential decay) will not remain true. The proof depends upon the process producing the jump.

The first process that comes to mind (Börner and Cohen, 1971) is that a flying saucer crashed onto the surface of the star, striking a glancing blow in such a direction as to speed it up. If we don't like flying saucers we can talk in terms of planets - masses on the order of $1 / 10$ that of the Earth are required in order to understand Vela. In this case $\Delta \Omega_{\mathrm{n}}=0$ and $\xi=I_{\mathrm{n}} t_{\mathrm{c}} / I \tau$, a quantity characteristic of the pulsar and depending in no way on the circumstances of the jump.

A second process in a neutron starquake (Ruderman, 1969b; Baym and Pines, 1971 j. It is not obvious what to take for $\Delta \Omega_{\mathrm{n}}$ here. Since the mass of the crust is small compared with that of the neutrons $\Delta \Omega_{\mathrm{n}} \ll \Delta \Omega_{\mathrm{c}}$ seems appropriate, leading to an identical conclusion. If $\Delta \Omega_{\mathrm{n}}$ is comparable to $\Delta \Omega_{\mathrm{c}}$ we would require their ratio to be independent of $\Delta \Omega_{\mathrm{c}}$ for the proof to hold.

A third process is a sudden change in the pulsar magnetosphere (Scargle and Pacini, 1971). Here one imagines a massive cloud of plasma to suddenly detach from the corotating magnetic field and escape to infinity. This has two effects. First, it reduces the moment of inertia of the charged particle system - an effect identical to that of a starquake, with the added attraction that $\Delta \Omega_{\mathrm{n}}$ is always zero. Secondly, the explosive release of plasma can produce an impulsive reaction torque on the star - an effect identical to that of a collision with a planet. Again, $\xi$ is constant in time.

Finally, consider a sudden transfer of angular momentum from neutrons to charges. Whether this might conceivably be accomplished will be discussed later but for now we 
simply note that typical values of $\left(\Omega_{\mathrm{n}}-\Omega_{\mathrm{c}}\right) / \Omega_{\mathrm{c}}$ amount to several percent. The neutron superfluid thus represents an enormous storehouse of 'excess' angular momentum relative to the charges. The transfer of even a small fraction of this excess could easily account for the observed jumps.

In this picture the neutrons slow down as the charges speed up and $I_{\mathrm{n}}\left(\Delta \Omega_{\mathrm{n}}\right)=$ $-I_{\mathrm{c}}\left(\Delta \Omega_{\mathrm{c}}\right)$. Then $\xi=t_{\mathrm{c}} / \tau$, again constant from one jump to the next. Note that this model would predict $Q=1$.

What is the observational situation? With regard to Vela we do not yet know the value of $\Delta\left|\Omega_{\mathrm{c}}\right|$ for the second jump. It does seem to be positive (Reichley and Downes, 1971). The situation with regard to the Crab pulsar is almost equally ambiguous. The Princeton group (Boynton et al., 1972) has published what seems to be the most complete analysis of its first jump. The only published analysis of its second jump (Lohsen, 1972) does not discuss the post-jump behavior in terms of an exponential period decay. The physical meanings of the values of $\xi$ derived from the two analyses are then quite different, and no comparison is possible. (If we neglect this difficulty and baldly compare values we find $\xi$ (first jump) $\cong 10 \times \xi$ (second jump).) It is fortunate that Lohsen's observations do provide a continuous string of data during the period immediately following the jump. Re-analysis of these data should provide a detailed check of the theory.

\subsection{Frictional heAting}

Cameron (unpublished) has added a further critical element to our picture of superfluid neutron stars. He noted that because the bulk of the mass of the star is slowed by frictional means, heat must be steadily dissipated in the process. Consider $1 \mathrm{~cm}^{3}$ of superfluid. The frictional force $f$ of (1) on this fluid element multiplied by $\left(v_{n}-v_{c}\right)$ is the rate that work is being done on the neutrons. All this work is dissipated as heat. The rate of dissipation is then

$$
\begin{aligned}
\dot{E}_{\text {heat }} & =\mathbf{f} \cdot\left(\mathbf{v}_{\mathrm{n}}-\mathbf{v}_{\mathrm{c}}\right) \\
& =(\text { torque })\left(\Omega_{\mathrm{n}}-\Omega_{\mathrm{c}}\right) \\
& =\varrho_{\mathrm{n}}(r \sin \theta)^{2} \Omega_{\mathrm{n}}\left(\Omega_{\mathrm{n}}-\Omega_{\mathrm{c}}\right) \mathrm{erg} \mathrm{cm}^{-3} \mathrm{~s}^{-1} .
\end{aligned}
$$

Neglecting the rotational energy in the charges the rate of loss of rotational energy is $\dot{\mathrm{E}}_{\text {rotation }} \cong \varrho_{\mathrm{n}}(r \sin \theta)^{2} \dot{\Omega}_{\mathrm{n}} \Omega_{\mathrm{n}} \mathrm{erg} \mathrm{cm}^{-3} \mathrm{~s}^{-1}$ so that

$$
\frac{\dot{E}_{\text {neat }}}{\dot{E}_{\text {rotation }}} \cong \frac{\Omega_{\mathrm{n}}-\Omega_{\mathrm{c}}}{\Omega_{\mathrm{n}}} \cong \frac{\Omega_{\mathrm{n}}-\Omega_{\mathrm{c}}}{\Omega_{\mathrm{c}}}
$$

Neutron stars are end points of stellar evolution. As such they are generally thought to possess no internal sources of energy. We now see that this is not so. If superfluid they are able to convert a fraction, given by (11), of their rotational energy into internal heat. (The mechanism outlined here is not the only way this can be done. Henriksen et al. (1972) have shown that rotational energy can also be dissipated into heat by the action of a steady wobble.) An analysis of where this takes place (Greenstein, 1971) 
vithin the star shows the frictional energy generation to be predominantly confined o a thin shell lying within the lower regions of the crust.

The rate of energy generation integrated throughout the star must equal the rate he star radiates energy. For internal temperatures $T \leqq 10^{8} \mathrm{~K}$ the cooling mechanism s predominantly photon emission from the surface. Confining attention to this case

$$
\begin{aligned}
4 \pi R^{2} \sigma T_{e}^{4} & =\int \mathrm{d}^{3} r \dot{E}_{\text {heat }} \\
& \cong \int \mathrm{d}^{3} r \varrho_{\mathrm{n}}(r \sin \theta)^{2} \dot{\Omega}_{\mathrm{c}}\left(\Omega_{\mathrm{n}}-\Omega_{\mathrm{c}}\right) \\
& \cong \int \mathrm{d}^{3} r \varrho_{\mathrm{n}}(r \sin \theta)^{2} \dot{\Omega}_{\mathrm{c}} \Omega_{\mathrm{c}} \varrho \tau / \varrho_{\mathrm{c}} t_{\mathrm{c}}
\end{aligned}
$$

where $R$ is the stellar radius, $\sigma$ the Stephan-Boltzmann constant and $T_{\mathrm{e}}$ the surface temperature. The right-hand side of (12) contains $\tau$, which is a function of $T$. If the relation between $T$ and $T_{\mathrm{e}}$ is known (12) becomes an equation which can be solved for the temperature. A rough treatment (Greenstein, 1971) yields

$$
T_{\mathrm{e}} \cong \frac{4 \times 10^{7}}{\left[p t_{y}^{2}\right]^{1 / 6}} \mathrm{~K},
$$

where $p$ is the pulsar period in seconds and $t_{\mathrm{y}} \equiv \mathrm{p} / \dot{\mathrm{p}}$ in years. This result gives the lowest surface temperature such a star can attain. Because of the crudeness of the approximations leading to (13), detailed predictions based upon it are subject to doubt. Keeping this in mind I wish to apply it to three specific examples.

Before doing so a general comment should be made. Before 1967 the way one searched for neutron stars was to search for blackbody X-ray emission from their surfaces. No such sources were ever found. Since the discovery of pulsars and their identification with rotating neutron stars this project has been largely dropped. I wish to emphasize that the evidence that pulsars are neutron stars, though strong, is indirect. It would be nice to find something direct. The discovery of a point source of blackbody X-ray emission at a position coinciding with that of a pulsar would constitute such evidence.

The first example I want to discuss is the closest pulsar, CP 0950. Equation (13) predicts its surface temperature to be $2 \times 10^{5} \mathrm{~K}$. The spectrum peaks at $0.1 \mathrm{keV}$ energies. The X-ray flux at this energy at the top of the Earth's atmosphere is $10^{-28}$ erg $\mathrm{cm}^{-2} \mathrm{~s}^{-1} \mathrm{~Hz}^{-1}$ (estimating the pulsar's distance from its dispersion measure and neglecting interstellar absorption). Such a flux, were it concentrated at $\mathrm{keV}$ energies, would be detectable. Because it is concentrated at $0.1 \mathrm{keV}$ energies the observations will be harder. Whether they are impossible is not clear.

The next example is the Crab pulsar. Its predicted temperature is $5 \times 10^{6} \mathrm{~K}$. The spectrum peaks at $2 \mathrm{keV}$ at which point the flux is $3 \times 10^{-27} \mathrm{erg} \mathrm{cm}^{-2} \mathrm{~s}^{-1} \mathrm{~Hz}^{-1}$. Such a flux is eminently detectable. Why, then, has it not been detected? Because it represents a small fraction (about $15 \%$ ) of the flux from the nebula as a whole at this 
energy. It seems that the only way to detect this flux would be to observe the nebula during a lunar occultation.

Let me describe a possible observation one might perform during such an occultation. As the limb of the moon sweeps across the nebula the received X-ray flux diminishes steadily. As the limb sweeps across the pulsar the flux decreases discontinuously. Of this sudden kink some is due to the obscuration of the X-ray pulsar. Suppose that one accurately knows the pulsar intensity. The difference between it and the observed kink represents the steady, non-pulsed, X-ray emission from the pulsar. If we assume this to be blackbody radiation from a neutron star we can take a canonical radius for the star and calculate its temperature. But there is far more we can do if the spectrum of the point source can be determined (by performing the above observation at several energies). If the spectrum is blackbody we will have strong reason to attribute it to thermal radiation from a neutron star. The spectrum determines the temperature. The received flux then determines the radius of the star. If this radius does not agree with expected neutron star radii we will have an interesting contradiction. If it does, and if we trust our models, the radius will determine the mass of the star. Now we can use the formula

$$
\dot{\mathrm{p}} \mathrm{p} \cong \frac{4 \pi^{2}}{c^{3}} B^{2} R^{6}
$$

to determine $B$, the pulsar's magnetic field.

(Equation [14] is true in those simple magnetospheric models such as those of Ostriker and Gunn (1969), and Goldreich and Julian (1969), which predict a braking index $N=3$. It is presumably not too badly off for the Crab, for which $N \cong 2.5$.) Finally, $\tau$, the relaxation time describing the post-speedup decay of the Crab pulsar, is known to be $\approx 4$ days. Theoretically, $\tau$ is a function of temperature and mass. A number of fruitful comparisons between theory and observation are possible here too. So, in one fell swoop, we would have gained an enormous amount of information.

I would not have wasted so much time on this were the next lunar occultation some time in the 1980's. In fact the next lunar occultation is only a year and a half away on March 29, 1974. From then on the Moon will occult the Crab every month till late 1975, a total of 20 occultations. Some details concerning the occultations are given in Table I. The years 1974-75 are going to present golden opportunities, not to return for something like a decade. I believe that reliable observations such as I have described could provide crucial data to the theoreticians. I hope they will be performed. So tell all your friends.

We already have one observational handle on the temperature of the Crab pulsar. This is the historic lunar occultation experiment of Bowyer èt al. (1964) which showed the bulk of the X-ray emission to be nebular in origin. This experiment just missed detecting the X-ray pulsar. At a temperature predicted by (13) the thermal radiation from the star at $2 \mathrm{keV}$ would be slightly higher than that from the pulsar. Thus, were its temperature significantly higher than $5 \times 10^{6} \mathrm{~K}$, thermal $X$-rays from the Crab 
neutron star would already have been detected. Again, this result neglects interstellar (or nebular) absorption.

The last example is the recently discovered X-ray source GX340+0 (Margon et al., 1971). This is the only source we know of whose spectrum has been definitely established to be blackbody. The observed temperature is $1.5 \times 10^{7} \mathrm{~K}$ - quite hot. From distance estimates $(4 \mathrm{kpc})$ and the observed flux the radius of the emitting body is found to be $8 \pm 4 \mathrm{~km}$. It looks exactly like a neutron star. How old is it?

We can get a cooling curve from (13) (Greenstein, 1972) if we assume a magnetospheric model. If we assume the approximate validity of those for which the braking index $N=3$ then $\mathrm{p} \dot{\mathrm{p}}$ is constant in time. Writing $t_{\mathrm{c}}=\mathrm{p}^{2} / \mathrm{p} \dot{\mathrm{p}}$ and noting that if $N=3$, $t_{\mathrm{c}}=2 t$ we can find the temperature in terms of the time:

$$
T_{\mathrm{e}} \cong \begin{gathered}
10^{8} \\
\chi^{1 / 12} t^{5 / 12} \\
\mathrm{~K}
\end{gathered}
$$

where $t$ is the age in years. Here $\chi$ has been defined to be $\mathrm{p} \dot{\mathrm{p}} \times 10^{15}$. In known pulsars $\chi$ ranges from 0.06 to 150 with a mean value of order unity. Specializing to the observed temperature of $\mathrm{GX} 340+0$ yields an age for this object of $125 \chi^{-1 / 5} \mathrm{yr}$, a period of $0.003 \chi^{2 / 5} \mathrm{~s}$ and a luminosity (rate of loss of rotational energy) of $\left(10^{40}\right.$ to $\left.10^{42}\right) \chi^{-3 / 5}$ ergs $\mathrm{s}^{-1}$. It makes sense to search carefully for a supernova remnant and a pulsar at the location of this source. If we assume the supernova remnant expands at $\approx 10^{3} \mathrm{~km}$ $\mathrm{s}^{-1}$ its angular diameter would be a few seconds of arc.

\section{TABLE I}

Forthcoming lunar occulatations of Crab Nebula * Adopted position (1950) $05^{\mathrm{h}} 31^{\mathrm{m}} 30^{\mathrm{s}} .5$

$$
+21^{\circ} 59^{\prime} 01^{\prime \prime}
$$

\begin{tabular}{|c|c|c|c|}
\hline Date & $\begin{array}{l}\text { Time of } \\
\text { Conjunction } \\
\text { (nearest hour) }\end{array}$ & $\begin{array}{l}\text { Elongation } \\
\text { of Moon } \\
\text { from Sun }\end{array}$ & Area of visibility \\
\hline 1974 & (hours) & (degrees) & \\
\hline March 29 & 22 & 77 & $\begin{array}{l}\text { Arctic and North Scandinavia at low } \\
\text { altitude }\end{array}$ \\
\hline April 26 & 04 & 50 & $\begin{array}{l}\text { Central and Northern Asia, Arctic, Alaska, } \\
\text { Canada and North USA. }\end{array}$ \\
\hline May 23 & 11 & 23 & $\begin{array}{l}\text { Northeast America, Greenland, Arctic, } \\
\text { North Scotland, Scandinavia, Finland, } \\
\text { Central Asia }\end{array}$ \\
\hline June 19 & 21 & 356 & $\begin{array}{l}\text { Northeast Asia, Arctic, Alaska, Canada, } \\
\text { Northeast of USA }\end{array}$ \\
\hline July 17 & 08 & 329 & $\begin{array}{l}\text { Northeast Canada, Greenland, Arctic, N. } \\
\text { Scotland, Scandinavia, Finland, Central } \\
\text { Asia }\end{array}$ \\
\hline Aug. 13 & 17 & 302 & $\begin{array}{l}\text { Japan, E. Asia, Alaska, Arctic, Greenland, } \\
\text { N. America }\end{array}$ \\
\hline Sept. 10 & 01 & 275 & $\begin{array}{l}\text { N. Africa, Europe (west at low altitude), } \\
\text { Central Asia, Japan }\end{array}$ \\
\hline
\end{tabular}


Table I (continued)

\begin{tabular}{|c|c|c|c|}
\hline Date & $\begin{array}{l}\text { Time of } \\
\text { Conjunction } \\
\text { (nearest hour) }\end{array}$ & $\begin{array}{l}\text { Elongation } \\
\text { of Moon } \\
\text { from Sun }\end{array}$ & Area of visibility \\
\hline Oct. 07 & 07 & 248 & $\begin{array}{l}\text { Central and N. America (but not White } \\
\text { Sands), N. Atlantic, S. and S. W. Europe, } \\
\text { N. Africa }\end{array}$ \\
\hline Nov. 03 & 12 & 221 & $\begin{array}{l}\text { Central Pacific, Hawaii, Central America } \\
\text { and north of S. America at low altitude }\end{array}$ \\
\hline Nov. 30 & 20 & 194 & $\begin{array}{l}\text { Central Africa (west at low altitude), } \\
\text { Arabia, India, S. E. Asia, northern cost of } \\
\text { Australia at very low altitude. }\end{array}$ \\
\hline Dec. 28 & 06 & 167 & $\begin{array}{l}\text { Central Pacific, Hawaii, Central and N. } \\
\text { America, north of S. America, central } \\
\text { Atlantic }\end{array}$ \\
\hline \multicolumn{4}{|l|}{1975} \\
\hline Jan. 24 & 17 & 140 & $\begin{array}{l}\text { Central Africa, Arabia, India, S. E. Asia, } \\
\text { northern coast of Australia at low altitude. }\end{array}$ \\
\hline Feb. 21 & 02 & 113 & $\begin{array}{l}\text { S. Pacific, S. America (east at low altitude), } \\
\text { Mexico }\end{array}$ \\
\hline March 20 & 10 & 86 & $\begin{array}{l}\text { Indian Ocean, East Indies, Australia, New } \\
\text { Zealand at low altitude }\end{array}$ \\
\hline April 16 & 15 & 60 & $\begin{array}{l}\text { E. coast of S. America at very low altitude, } \\
\text { S. Atlantic, S. Africa and Madagascar }\end{array}$ \\
\hline May 13 & 21 & 33 & S. Pacific, S. America, Antarctic \\
\hline June 10 & 04 & 6 & Indian Ocean, Australia, New Zealand \\
\hline July 07 & 13 & 339 & $\begin{array}{l}\text { S. America, Antarctic, very near Cape of } \\
\text { Good Hope at low altitude }\end{array}$ \\
\hline Aug. 03 & 23 & 312 & Australia, New Zealand, Antarctic \\
\hline Aug. 31 & 08 & 285 & Tip of South America, Antarctic \\
\hline
\end{tabular}

* Source: L. V. Morrison, H. M. Nautical Almanac Office, Royal Greenwich Observatory, Herstmonceux Castle, England.

I am grateful to Dr. Morrison for furnishing me with this information.

\section{The Most Complicated Possible Model of Superfluid Neutron Star}

None of the results we have derived so far are rigorous. Indeed, they may be off by orders of magnitude. Why?

They were derived under the assumption that $\varrho \tau / \varrho_{c}$ is constant. In fact it varies by orders of magnitude throughout the star. Thus the right-hand side of (3) is a strong function of density. Since $\Omega_{\mathrm{c}}$ cannot be then $\Omega_{\mathrm{n}}$ must be. Therefore the neutrons are in a state of differential rotation. This does not sound particularly horrible. Were the neutrons to form an ordinary fluid it would not be. But because they form a superfluid it makes all the difference in the world.

\subsection{SuPERfLUID TURBULENCE (Greenstein, 1970).}

A rotating superfluid must contain vortex lines. These lines are in a state of tension 
and would like to form a uniform array parallel to the rotation axis. The tension in a line is finite, however, so that a sufficiently strong shearing force can disturb this state. Let us first completely neglect line tension. At every point within the superfluid the lines move with essentially the same velocity as the superfluid (the relative velocity between line and fluid, determined by the Magnus effect, amounts to $\approx 10^{-4} \mathrm{~cm} \mathrm{~s}^{-1}$ ). Different regions of the superfluid rotate with different angular velocities. Each vortex line is therefore steadily lengthening and twisting about the rotation axis (in the same way that a rubber band, stretched between two cars moving with different speeds, steadily lengthens). Every time one region of the superfluid has lapped another once, the vortex lines passing between them have wrapped once more about the star. Eventually the wrapping is very tight. Along the equator lines of opposite senses are brought near each other. This situation is unstable and eventually two opposing lines will be brought sufficiently close to reconnect, forming a vortex ring and a shorter vortex line. The ring migrates away, the line steadily lengthens, new rings form and the process continues. As a given ring migrates about it collides with others and with lines, exciting vortex waves in them. Eventually a ring will be broken into two smaller rings. Feynman (1955) speculates that the ring of smallest possible diameter is a roton. If so, the breaking up of large rings leads in the end to the heating of the fluid. The whole process constitutes the superfluid version of the dissipation of velocity differentials into heat. In the steady state the superfluid contains tightly wrapped vortex lines, no longer parallel to the rotation axis, plus large numbers of rings. The distribution of lines fluctuates irregularly. The superfluid velocity field, determined by the distribution of lines, also fluctuates irregularly. This state is a superfluid version of fully developed turbulence. The turbulence is microscopic with typical eddy sizes being the distance between lines $\left(\lesssim 10^{-2} \mathrm{~cm}\right)$.

Recall that $\tau$, the relaxation time describing the coupling between charges and superfluid, depends on scattering processes taking place within the normal cores of vortex lines (and rings). It is therefore inversely proportional to the total length of vortex line present. If the lines are greatly lengthened $\tau$ is proportionately shortened.

It is difficult to estimate the magnitude of this effect. A given line must wrap very many times about the star before opposing ends come sufficiently close to reconnect and form rings. These rings can themselves survive for long periods of time before being broken apart. In the absence of detailed knowledge it seems safe to say that $\tau$ is decreased by this effect by orders of magnitude. The problem is we don't know by how many orders of magnitude!

Of course vortex lines are in a state of tension. This tension acts to 'rigidify' the rotation of the superfluid. Let us begin with a uniformly rotating superfluid and ask if line tension is sufficient to maintain this state. In uniform rotation each line is parallel to the rotation axis. The scattering force $f$ acting upon it, given by (1), varies by orders of magnitude along its length. The line will be able to maintain the state of uniform rotation if its tension force $F_{\text {tension }}$ is greater than the total scattering force $F_{\text {scattering }}$ (equal to $f$ integrated along the line) acting upon it. Numerically 


$$
\begin{aligned}
F_{\text {tension }} & =\pi \varrho_{\mathrm{n}}\left(\frac{\hbar}{2 m_{\mathrm{n}}}\right)^{2} \ln \left(\frac{b}{a}\right) \\
& \cong 5 \times 10^{8} \text { dyne }
\end{aligned}
$$

at $\varrho_{\mathrm{n}}=10^{14} \mathrm{~g} \mathrm{~cm}^{-3}$. Here $m_{\mathrm{n}}$ is the mass of the neutron, $b$ the distance between vortex lines and $a$ the vortex core radius. We can find $F_{\text {scattering }}$ as follows. In the steady state it adjusts itself until the total torque on the neutrons is sufficient to slow them down at the same rate as the charges. Thus

Also

$$
\text { torque }=I_{\mathrm{n}} \dot{\Omega}_{\mathrm{n}} \cong M R^{2} \dot{Q}_{\mathrm{c}}
$$

Thus

$$
\begin{aligned}
\text { torque } & \cong\left(\begin{array}{c}
\text { number of } \\
\text { lines in } \\
\text { the star }
\end{array}\right) R F_{\text {scattering }} \\
& \cong\left(4 \times 10^{15} \Omega_{\mathrm{c}}\right) R R_{\text {scattering }} .
\end{aligned}
$$

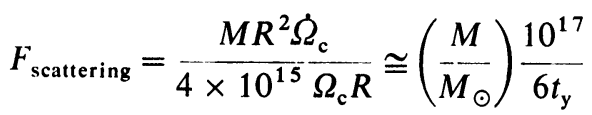

$\left(t_{\mathrm{y}} \equiv\left|\Omega_{\mathrm{c}} / \dot{\Omega}_{\mathrm{c}}\right|\right.$ in years). The condition $F_{\text {tension }}>F_{\text {scattering }}$ then yields

$$
t_{\mathrm{y}}>3\left(\frac{M}{M_{\odot}}\right) \times 10^{7} \mathrm{yr} .
$$

So all pulsars whose characteristic age is less than $\approx 3 \times 10^{7} \mathrm{yr}$ will exhibit superfluid turbulence.

There may be a flaw in this proof. A uniform vortex lattice exerts forces that cannot be understood in terms of the properties of isolated lines. These forces are generally (see, e.g., Fetter and Stauffer, 1970) thought to be far weaker than tension forces. Ruderman (1972), however, has argued against this point of view. He will briefly describe his arguments elsewhere in these proceedings.

\subsection{HYDRODYNAMICS OF THE SUPERFLUID}

If vortex tension is unable to enforce rigid body rotation we are left with the task of finding out what the superfluid is doing. It is well known that fully developed turbulence possesses a turbulent kinematic viscosity $v=($ mean velocity in an eddy) $\times($ mean diameter of an eddy). The velocity field of a superfluid about a vortex line is $v=n h / 2 m_{\mathrm{n}} r$ where $n=1,2,3 \ldots$. Therefore, $v=n h / 2 m_{\mathrm{n}}$. This estimate is in agreement with observed properties of the turbulent flow of superfluid helium (Vinen, 1961). The existence of this viscosity will result in the formation of a boundary layer just below the base of the crust in which the superfluid corotates with the crust.

The existence of this boundary layer, produced by turbulent viscosity, fulfills the conditions required for Eckman pumping to begin. This process spins down the super- 
fluid in a time

$$
t_{\mathrm{E}} \approx R / \sqrt{v \Omega_{\mathrm{n}}} \approx 1 / \sqrt{n \Omega_{\mathrm{c}} \mathrm{yr} .}
$$

This time scale (one month for the Crab) is always short compared with $t_{\mathrm{c}}$. The neutron superfluid, then, will almost exactly corotate with the crust. Thus the dominant interaction between superfluid and charges may well be classical (viscous) in nature. Whether this is so depends on whether the turbulence develops sufficiently fully for such ideas to be applicable. There is a further problem. Are we correct in regarding the crust as 'containing' the superfluid? It may be that vortex lines interact with the crust quite weakly. If so, no boundary layer would form and, though viscous, the superfluid would not exhibit Eckman pumping.

If not, what does it do? The following ideas are exceedingly tentative. If we look at the rotation curve obtained from (3) we find two regions in the star in which $\Omega_{\mathrm{n}}$ decreases outwards. These regions coincide with those in which the superfluid energy gap decreases with decreasing density, i.e., at densities between $10^{14}$ and $10^{15} \mathrm{~g} \mathrm{~cm}^{-3}$, and at densities $<2 \times 10^{13} \mathrm{~g} \mathrm{~cm}^{-3}$. In these two regions $\Omega_{\mathrm{n}}$ may be decreasing outwards sufficiently rapidly for the quantity $r^{2} \Omega_{\mathrm{n}}$ to decrease outwards. If it does the flow is hydrodynamically unstable (the Rayleigh instability) towards the development of classical turbulence. Because only the neutrons undergo these motions there is no composition gradient acting to stabilize the flow.

Suppose the fluid is turbulent in this classical sense. Now the turbulent eddies are macroscopic. Perhaps the superfluid resembles a pan of boiling water. We know that such a pan occasionally spits up a blob of water into the air. Suppose the superfluid lying within the inner crust does this. The blob will penetrate into the outer crust and lodge there -- but the superfluid is rotating more rapidly than the crust. Therefore, the blob will impart its excess angular momentum to the crust, and will suddenly speed it up.

Should we identify this process with the observed intermittent large period jumps or with the smaller scale continuous fluctuations in the pulse repetition rate that the Princeton group (Boynton et al., 1972) claims to be random in nature? It is hard to be sure. Even the rotation curve derived from (3) cannot be correct, for (3) neglects strictly hydrodynamical forces acting on the superfluid. A rigorous analysis of the stability of the superfluid flow should begin from the full equations, averaged over many vortex lines, of superfluid hydrodynamics. For now we are only able to make a plausibility argument - that it seems reasonable that in those regions in which the coupling between neutrons and charges is weakest $\Omega_{\mathrm{n}}$ should be greatest. If so we would expect instabilities to develop.

I should emphasize that the problems we need to solve in order to understand what the superfluid is doing are soluble - an unusual state of affairs in neutron star physics. They are all problems in classical hydrodynamics (vortex lines are nineteenth century objects). We do not need to invent any new physics. It may even prove possible to exploit the strong resemblance between the neutron superfluid and superfluid helium to design laboratory experiments with which to gain insight into these phenomena. 


\section{References}

Baym, G. and Pines, D.: 1971, Ann. Phys. N.Y. 66, 816.

Baym, G., Pethick, C., Pines, D., and Ruderman, M.: 1969, Nature 224, 872.

Börner, G. and Cohen, J.: 1971, Nature Phys. Sci. 231, 146.

Bowyer, S., Byram, E. T., Chubb, T. A., and Friedman, H.: 1964, Science 146, 912.

Boynton, P. E., Groth, E. J., Hutchinson, D. P., Nanos, G. P., Partridge, R. B., and Wilkinson, D. T.: 1972, Astrophys. J. 175, 217.

Cameron, A. G. W.: 1970, Ann. Rev. Astron. Astrophys. 8, 179.

Fetter, A. and Stauffer, B.: 1970, Nature 227, 584.

Feynman, R.: 1955, in C. J. Gorter (ed.), Prog. Low Temperature Phys. 1, 17.

Goldreich, P. and Julian, W.: 1969, Astrophys. J. 157, 869.

Greenstein, G.: 1970, Nature 227, 791.

Greenstein, G.: 1971, Nature Phys. Sci. 232, 117.

Greenstein, G.: 1972, Nature Phys. Sci. 238, 71.

Greenstein, J. L.: 1969, Comments Astrophys. Space Phys. $1,62$.

Henriksen, R. N., Feldman, P. A., and Chau, W. Y.: 1972, Astrophys. J. 172, 717.

Lohsen, E.: 1972, Nature 236, 70.

Margon, B., Bowyer, S., Lampton, M., and Cruddace, R.: 1971, Astrophys. J. Letters 169, L45.

Ostriker, J. P. and Gunn, J. E.: 1969, Astrophys. J. 157, 1395.

Pines, D.: 1970, in E. Kanda (ed.), Proc. of the 12th International Conference on Low Temperature Phys.

Reichley, P. and Downes, G.: 1971, Nature Phys. Sci. 234, 48.

Ruderman, M.: 1969a, J. Phys. 30, C3-152.

Ruderman, M.: 1969b, Nature 223, 597.

Ruderman, M.: 1972, Ann. Rev. Astron. Astrophys. (in press).

Scargle, J. and Pacini, F.: 1971, Nature Phys. Sci. 232, 144.

Vinen, W. F.: 1961, in Prog. Low Temperature Phys. 3.

\section{DISCUSSION}

Ruderman: When the force along an isolated superfluid vortex line varies by more than the tension in the line, then the vortex line will behave as described by Prof. Greenstein. It will bend and twist in response to the external force. But an array of vortex lines can behave quite differently and the criterion that if an isolated line twists in a complicated way, then an array will likely become turbulent is generally not valid. From the vortex line point of view a group of $N$ closely spaced vortices has $N^{2}$ times the tension of a single vortex but only $N$ times the differential force: the array is very much stiffer relative to the impressed force.

A uniformly rotating neutron star superfluid is described by a dense parallel vortex array of spacing $b \sim 10^{-2} \mathrm{~cm}$. The scale of spatial variation of the differential torques on the neutron star superfluid is of order $R \sim 10^{6} \mathrm{~cm}$ so that $b / R \sim 10^{-8}$. The limit $b / R \rightarrow 0$, almost reached here, should be the same as that in which $\hbar \rightarrow 0$, i.e. classical hydrodynamics. This classical problem, the response of a uniformly rotating gravitating, compressible, nonviscous fluid in an axially symmetric container to a paraxial torque which varies with position but not angle $(\phi)$, can be solved. The results (an extension of the Taylor-Proudman theorem) show a uniform $\phi$-directed acceleration on each coaxial cylinder together with a slow circulation in the planes of constant $\phi$ (i.e. planes through the symmetry axis). In the vortex line description, the vortex lines remain almost rigid and parallel to the rotation axis. The only change with time is a motion which changes their number density so that this density remains proportional to the classical $\nabla \times \mathbf{v}$.

The classical instability which can occur when the angular momentum per unit mass decreases with increasing radius corresponds to $\nabla \times \mathbf{v}$ changing sign. In the superfluid vortex picture, where $\nabla \times \mathbf{v}$ vanishes so does the vortex line density and, consequently, so does the torque upon the fluid in that region. Thus the torque would not convert a stable rotating fluid into an unstable one whatever its spatial distributions. (A sign reversal in $\nabla \times \mathbf{v}$ cannot be obtained merely by moving vortex lines around but would need the spontaneous creation of oppositely directed lines in the body of the fluid.) 
Bethe: It seems that opposite statements have been made in the talk by Greenstein and in the discussion of it. Can someone elucidate what we should believe?

Greenstein: Don't believe anything quite yet. The problem with my approach is that I have considered the properties of an isolated vortex line. In fact the vortices form a lattice because there are long-range interactions between them. These interactions are very difficult to understand rigorously. The problem with Ruderman's approach is that it has not yet been formulated sufficiently fully to decide whether or not it represents a satisfactory treatment.

Stauffer has pointed out that, because the Tkachenko vortex lattice has a finite shear modulus, the system may split up into a few cylindrical regions, each rotating rigidly (if Ruderman's argument is correct). 